\title{
A Lower Global Lung Ultrasound Score Is Associated with Higher Likelihood of Successful Extubation in Invasively Ventilated COVID-19 Patients
}

\author{
Charalampos Pierrakos, ${ }^{1,2 \star}$ Arthur Lieveld, ${ }^{3} \dagger$ Luigi Pisani, ${ }^{1,4,5} \dagger$ Marry R. Smit, ${ }^{1} \dagger$ Micah Heldeweg, ${ }^{3} \dagger$ Laura A. Hagens, ${ }^{1}$ \\ Jasper Smit, ${ }^{3}$ Mark Haaksma, ${ }^{3}$ Lars Veldhuis, ${ }^{3}$ Robin Walburgh Schmidt, ${ }^{3}$ Giacomo Errico, ${ }^{4,6}$ Valentina Marinelli, ${ }^{7}$ \\ Rachid Attou, ${ }^{2}$ Cristina E. David, ${ }^{2}$ Claudio Zimatore, ${ }^{1,7}$ Francesco Murgolo, ${ }^{7}$ Salvatore Grasso, ${ }^{7}$ Lucia Mirabella, ${ }^{6}$ \\ Gilda Cinnella, ${ }^{6}$ David De Bels, ${ }^{2}$ Marcus J. Schultz, ${ }^{1,5,8}$ Pieter-Roel Tuinman, ${ }^{3,9}$ and Lieuwe D. Bos ${ }^{1}$ \\ ${ }^{1}$ Department of Intensive Care and Laboratory of Experimental Intensive Care and Anesthesiology, Amsterdam University Medical Centers- \\ AMC, Amsterdam, The Netherlands; ' ${ }^{2}$ Department of Intensive Care, Brugmann University Hospital, Université Libre de Bruxelles, Belgium; \\ ${ }^{3}$ Department of Intensive Care and Acute Internal Medicine, Amsterdam University Medical Centers - VUMC, Amsterdam, The Netherlands; \\ ${ }^{4}$ Department of Anesthesia and Intensive Care, Miulli Regional Hospital, Acquaviva delle Fonti, Italy; ${ }^{5}$ Mahidol-Oxford Tropical Medicine \\ Research Unit, Mahidol University, Bangkok, Thailand; ${ }^{6}$ Department of Anesthesia and Intensive Care, Foggia University Hospital, University of \\ Foggia, Italy; ${ }^{7}$ Intensive Care Unit, Department of Emergency and Organ Transplantation, University of Bari, Bari, Italy; ${ }^{8}$ Nuffield Department of \\ Medicine, University of Oxford, United Kingdom; ${ }^{9}$ ALIFE, Amsterdam Leiden IC Focussed Echograpy, Amsterdam, The Netherlands
}

\begin{abstract}
Lung ultrasound (LUS) can be used to assess loss of aeration, which is associated with outcome in patients with coronavirus disease 2019 (COVID-19) presenting to the emergency department. We hypothesized that LUS scores are associated with outcome in critically ill COVID-19 patients receiving invasive ventilation. This retrospective international multicenter study evaluated patients with COVID-19-related acute respiratory distress syndrome (ARDS) with at least one LUS study within 5 days after invasive mechanical ventilation initiation. The global LUS score was calculated by summing the 12 regional scores (range 0-36). Pleural line abnormalities and subpleural consolidations were also scored. The outcomes were successful liberation from the ventilator and intensive care mortality within 28 days, analyzed with multistate, competing risk proportional hazard models. One hundred thirty-seven patients with COVID-19-related ARDS were included in our study. The global LUS score was associated with successful liberation from mechanical ventilation (hazard ratio [HR]: $0.9195 \%$ confidence interval [Cl] 0.87-0.96; $P=0.0007$ ) independently of the ARDS severity, but not with 28 days mortality (HR: 1.03; 95\% Cl 0.97-1.08; $P=0.36$ ). Subpleural consolidation and pleural line abnormalities did not add to the prognostic value of the global LUS score. Examinations within 24 hours of intubation showed no prognostic value. To conclude, a lower global LUS score 24 hours after invasive ventilation initiation is associated with increased probability of liberation from the mechanical ventilator COVID-19 ARDS patients, independently of the ARDS severity.
\end{abstract}

\section{INTRODUCTION}

Coronavirus disease 2019 (COVID-19) is responsible for hundreds of thousands of deaths worldwide, and this number is still rapidly increasing. ${ }^{1}$ Respiratory failure is the most common and severe complication of COVID-19, and bilateral and multilobar infiltrates can progress rapidly over the first few days of illness. ${ }^{2}$ Approximately $5 \%$ of hospitalized patients require admission to an intensive care unit (ICU), mainly for invasive mechanical ventilation. ${ }^{3}$ There is a high variability in the reported mortality across invasively ventilated COVID-19 patients. ${ }^{4-6}$ The severity of loss of aeration, typically assessed by chest computed tomography (CT) scan, has been related to outcomes in hospitalized COVID19 patients, ${ }^{7-9}$ but CT-scan capacity is limited and may not be available in resource-limited settings.

Lung ultrasound is a bedside, radiation-free, low-cost diagnostic imaging tool that can be used for assessing lung aeration and parenchymal abnormalities. ${ }^{10}$ The global lung ultrasound score (LUS) quantifies lung aeration by translating LUS patterns into a numerical score across 12 lung regions and summing the results. ${ }^{11,12}$ Previous studies have shown a correlation between LUS and severity of acute

${ }^{*}$ Address correspondence to Charalampos Pierrakos, Department of Intensive Care, Brugmann University Hospital, Université Libre de Bruxelles, Brussels. Belgium. E-mail: charalampos_p@hotmail. com

†These authors contributed equally to this work. respiratory distress syndrome (ARDS),$^{13}$ and with mortality in invasively ventilated critically ill patients. ${ }^{14}$ LUS has previously been performed in a general population of COVID-19 patients outside the hospital, ${ }^{15}$ presenting to the emergency department (ED) $)^{16-18}$ and on the general wards ${ }^{19-26}$ and has been found to be related to adverse outcomes including the need of invasive ventilation. Nevertheless, the role of LUS in evaluating the severity of patients after initiation of invasive ventilation is much less certain. ${ }^{27}$ Evaluating lung disease severity with LUS may be important for ICU resource planning, especially in settings where resources are restricted, as well as for personalized ventilatory approach. ${ }^{28}$

The aim of this study is to assess the association between global LUS score and outcome, specifically defined as liberation from the ventilator and survival in critically ill COVID-19 patients under invasive ventilation. We hypothesized the global LUS score to have prognostic value in invasively ventilated COVID-19 patients independently of ARDS severity.

\section{METHODS}

Design. This is an international multicenter cohort study. We retrospectively reviewed patients with reverse transcriptase polymerase chain reaction confirmed COVID-19 under invasive ventilation in ICUs of four hospitals in three countries: the Brugmann University Hospital Brussels (Brussels, Belgium), the Miulli Regional Hospital (Acquaviva delle Fonti, Italy), and the Amsterdam University Medical Centers, locations AMC and VUMC, between February 2020 and 
December 31, 2020. LUS studies were performed as part of routine practice and were executed by experienced ultrasonographers $(N=10)$ who performed at least 50 systematic lung ultrasound exams before.

Ethics. Ethical approval for this study was provided by the ethical committees of each hospital (Brugmann University Hospital No. CE 2020/136; Azienda OspedalieroUniversitaria Policlinico di Bari 0030638/22/04/2020; Amsterdam UMC location AMC W18_311; Amsterdam UMC location VUMC 2020.011).

Patients. Patients were included if they received an LUS within first 5 days after invasive ventilation initiation but before extubation. Patients who received a LUS while supported with extracorporeal membrane oxygenation were excluded. Only the first available examination of LUS was used for the analysis.

Data collected. The following data were extracted from chart: demographic characteristics, APACHE II and SOFA score on admission, vital signs, and ventilator and blood gas parameters on the day of LUS examination.

The protocol for LUS. Lung ultrasound examination was performed with the available equipment at the COVID ICUs of the participating hospitals. The following machines were available: MyLab Five ultrasound machine with a convex probe (Esaote Spa, Genova, Italy), a Vivid S5 with curvilinear probe (General Electric Healthcare, Chicago, IL), a LOGIQ E with a linear probe (GE Healthcare, Milwaukee, WI), and a Sonosite Edge II (Fujifilm Sonosite, Bothell, WA).

The 12-region technique was used in all examinations in which ultrasound was performed on six areas on each side of the chest: two ventral regions, two lateral regions, and two posterolateral regions. ${ }^{29}$ The aeration pattern observed in each region was scored from 0 to 3 according to the LUS aeration score as follows: $0=\mathrm{A}$ pattern with $\leq 2 \mathrm{~B}$ lines; $1=>2$ separated $B$ lines that cover $\leq 50 \%$ of the pleural line; $2=\mathrm{B}$ lines that cover $>50 \%$ of the pleural line; or 3 = lung consolidation. In theory, the global LUS score can range from 0 (normal aeration in all regions) to 36 (severe abnormal aeration in all regions). ${ }^{30}$ Presence of subpleural consolidations and abnormal pleural line was also assessed offline in each field using saved ultrasound clips. Thickening and fragmentation of the pleural line and the finding of subpleural consolidations do not currently contribute to the LUS aeration score but are often found in patients with COVID-19 infection. Therefore, we assessed the number of fields with pleural line abnormalities and the number of fields with subpleural consolidations in a separate analysis. Pleural line abnormalities were defined as any deviation from the normally appearing thin, smooth and continuous hyperechoic line (Figure 1). ${ }^{29}$ Subpleural consolidations were defined as one or more echo poor regions juxtaposed to the pleural line, which were not large enough to be scored as a tissue-like pattern or "lobar consolidation" in the lung aeration score (Figure 1). ${ }^{30}$

Outcomes. The primary outcomes were the risks for successful liberation from invasive ventilation and intensive care mortality up to 28 days.

Statistical analysis. No formal power analysis was performed for this exploratory analysis. Rather, all patients that fulfilled the inclusion and exclusion criteria were included. Demographic, clinical, and outcome variables were presented as percentages for categorical variables and as medians with interquartile ranges (IQR) for continuous variables.
The association of LUS with outcomes was analyzed with multistate, competing risk proportional hazard models as described in the survival package via the compete function in $R$. Risks were estimated for successful extubation and mortality and compared with persistent intubation (reference category). Follow-up was censored after 28 days. Two sensitivity analysis were performed for the following predefined subgroups: 1) severity of ARDS according to $\mathrm{PaO}_{2} / \mathrm{FiO}_{2}$ based on cutoffs described in the Berlin definition ${ }^{31}$ and 2) days of invasive ventilation before LUS examination (exam on day 0 , day 1 , day $2-3$, day $4-5$ ).

Receiver operating characteristic (ROC) curve analysis was used to derive the prognostic discriminatory performance of global LUS in determining successful liberation from invasive ventilation and mortality at day 28 . On the basis of the calculated area under the ROC curve (AUROCC), the prognostic accuracy was interpreted as follows: excellent between 0.9 and 1 , good between 0.8 and 0.89 , fair between 0.7 and 0.79 , poor between 0.6 and 0.69 , and very poor between 0.5 and $0.59 .^{32}$ AUROCCs were compared using the DeLong test. Two cutoffs were defined: one with a high sensitivity of $>80 \%$ for poor outcome (composite of persistent mechanical ventilation at day 28 or mortality; selecting for a good negative predictive value) and one with a high specificity of above $80 \%$ for poor outcome (selecting for a good positive predictive value). The analysis was repeated using the three categories resulting from these cutoffs. All analyses were performed in $R$ through the R-studio interface (www.r-project.org, $R$ version 3.3.1). A $P$ value $<0.05$ was considered significant.

\section{RESULTS}

Patients. The study included 137 patients. Patient characteristics are presented in Table 1. At day 28, 53 patients (38\%) were successfully extubated, 64 patients $(47 \%)$ had died, and 20 patients (15\%) were still receiving invasive mechanical ventilation. Compared with the patients who failed to be extubated within 28 days, patients who were successfully extubated had a higher $\mathrm{PaO}_{2} / \mathrm{FiO}_{2}$ of $148 \mathrm{~mm}$ $\mathrm{Hg}$ (IQR: 115-173) versus $113 \mathrm{~mm} \mathrm{Hg}$ (IQR: 98-153, $P=0.013)$ and a lower global LUS score of 18 (IQR: 15-23) versus 21 points (IQR: 18-24, $P=0.005$ ).

Association between global LUS score and outcome. The global LUS score was associated with successful liberation from invasive ventilation (hazard ratio [HR]: 0.91; 95\% confidence interval $[\mathrm{Cl}]$ : $0.87-0.96 ; P=0.0007)$ but not with 28 days mortality (HR: 1.03 ; 95\% $\mathrm{Cl}$ : $0.97-1.08 ; P=0.36$ ) in competing risk analysis. However, the prognostic capacity of the global LUS sore for successful liberation from the ventilator at day 28 and mortality was poor (AUROCC of 0.65 ; 95\% Cl 0.54-0.74; AUROCC of $0.63 ; 95 \% \mathrm{Cl} 0.53-0.72$, respectively). The optimal cutoff for $>80 \%$ sensitivity for the combined endpoint of persistent mechanical ventilation or death at day 28 was 17 , whereas it was 24 for $>80 \%$ specificity (Table 2). The corresponding HRs for the probability of being liberated from the ventilator compared with a score of 17 or below (low risk group, $N=41)$, were $0.47(95 \% \mathrm{Cl}$ : $0.26-0.85 ; P=0.01)$ for the patients with LUS score 18 to 24 (intermediate risk group, $N=71)$ and $0.37(95 \% \mathrm{Cl}$ : $0.17-0.79 ; P=0.01$ ) for the patients with LUS above 24 (high risk group, $N=25$; Figure 2). Only patients with a global LUS 
TABLE 1

Characteristics of patients with COVID-19 disease examined with lung ultrasound

\begin{tabular}{|c|c|c|c|}
\hline & $\begin{array}{l}\text { Successful liberation of mechanical } \\
\text { ventilation and alive at } 28 \text { days }\end{array}$ & $\begin{array}{l}\text { Still intubated or } \\
\text { deceased at } 28 \text { days }\end{array}$ & $P$ value \\
\hline Number of patients & 53 & 84 & \\
\hline Day of LUS & $1(0-2)$ & $1(0-2)$ & 0.71 \\
\hline \multicolumn{4}{|l|}{ Demographics } \\
\hline Age, years & $61(54-68)$ & $71(62-76)$ & $<0.01$ \\
\hline Sex, female & $32(61)$ & $46(54)$ & 0.59 \\
\hline APACHE II & $13(12-17)$ & $14(12-22)$ & 0.21 \\
\hline SOFA score & $7(5-8)$ & $8(5-10)$ & 0.06 \\
\hline Global LUS score & $18(15-23)$ & $21(18-24)$ & $<0.01$ \\
\hline Subpleural consolidations, fields per patient & $3(1-5)$ & $5(2-6)$ & 0.01 \\
\hline Pleural line abnormalities, fields per patient & $3(1-5)$ & $3(1-5)$ & 0.91 \\
\hline \multicolumn{4}{|l|}{ Biology } \\
\hline D-dimers, $\mathrm{ng} / \mathrm{mL}$ & $1,440(873-3,875)$ & $2,326(1720-4,063)$ & 0.03 \\
\hline $\mathrm{CRP}, \mu \mathrm{g} / \mathrm{mL}$ & $77(40-159)$ & $108(21-172)$ & 0.81 \\
\hline \multicolumn{4}{|l|}{ Ventilation parameters } \\
\hline \multicolumn{4}{|l|}{ Ventilation mode } \\
\hline Volume controlled & $20(38)$ & $36(42)$ & 0.59 \\
\hline Pressure controlled & $17(32)$ & $30(36)$ & 0.71 \\
\hline Pressure support & $16(30)$ & $18(22)$ & 0.31 \\
\hline Tidal volume, $\mathrm{ml}$ & $425(378-484)$ & $418(390-460)$ & 0.71 \\
\hline Tidal volume, $\mathrm{ml} / \mathrm{kg}$ PBW & $6.3(5.8-6.9)$ & $6.3(5.6-6.5)$ & 0.75 \\
\hline PEEP set, $\mathrm{cm} \mathrm{H}_{2} \mathrm{O}$ & $10(8-12)$ & $10(8-12)$ & 0.81 \\
\hline Pplat, $\mathrm{cm} \mathrm{H} \mathrm{H}_{2} \mathrm{O}$ & $22(19-26)$ & $24(20-27)$ & 0.21 \\
\hline Driving Pressure & $12(11-17)$ & $14(10-17)$ & 0.55 \\
\hline Static Compliance & $35(26-44)$ & $31(24-44)$ & 0.55 \\
\hline $\mathrm{SpO}_{2}, \%$ & $94(93-96)$ & $95(94-97)$ & 0.83 \\
\hline $\mathrm{FiO}_{2}$ & $55(50-70)$ & $64(55-80)$ & $<0.01$ \\
\hline $\mathrm{PaO}_{2} / \mathrm{FiO}_{2}$ & $148(115-173)$ & $113(98-153)$ & 0.01 \\
\hline \multicolumn{4}{|l|}{ Complications/procedures } \\
\hline VAP & $11(21)$ & $59(71)$ & $<0.01$ \\
\hline ECMO & $0(0)$ & $4(5)$ & 0.15 \\
\hline Tracheostomy & $5(9)$ & 19 (22) & 0.06 \\
\hline Death (28 days) & $0(0)$ & $64(76)$ & \\
\hline
\end{tabular}

Data are presented as mean ( \pm SD) median (interquartile range) or number (\%). APACHE = Acute Physiology and Chronic Health Evaluation; ECMO = extracorporeal membrane oxygenation; $\mathrm{FiO}_{2}=$ fraction of inspired oxygen; ICU = intensive care unit; LUS = lung ultrasound; $\mathrm{PaO}_{2}=$ arterial oxygen tension; $\mathrm{PBW}=$ predicted body weight; PEEP = positive end-expiratory pressure; Pplat = plateau pressure; SOFA = Sequential Organ Failure Assessment; $\mathrm{SpO}_{2}=$ peripheral pulse oxymetric saturation; VAP = ventilator associated pneumonia.

score of 24 and above had an increased probability of death (HR: 2.3; 95\% Cl: 1.08-4.8; $P=0.03$ ) compared with a score of 17 or below. Adding subpleural consolidations or pleural line abnormalities did not improve the prognostic capacity for successful extubation or mortality at day 28 (Table 2).

Subgroup analyses. When patients were classified according to ARDS severity, 11 patients (9\%) had mild, 85 patients (68\%) had moderate, and 29 (23\%) had severe ARDS. For 12 patients $\mathrm{PaO}_{2} / \mathrm{FiO}_{2}$ was missing. The global LUS score was associated with the probability of being liberated from the ventilator while alive at 28 days after intubation independently of ARDS severity (Figure 3). There was no evidence for variation of global LUS association with outcome according to the categories of $\mathrm{P}_{\mathrm{a}} \mathrm{O}_{2} / \mathrm{FiO}_{2}$ (no interaction; $P=0.49$ ).

The majority of the patients were examined within 24 hours after invasive ventilation initiation (61 patients, 44\%), whereas 39 patients (28\%) were examined between 24 and 48 hours, 24 patients (17\%) between 48 and 96 hours, and 13 patients $(9 \%)$ between 96 and 120 hours after start of invasive ventilation. There was evidence variation of global LUS score according to the time of examination (interaction term, $P=0.036)$. When the examination was performed within 24 hours after intubation, the global LUS score was not associated with the probability of successful extubation in the first 28 days (Figure 3 ).

\section{A}

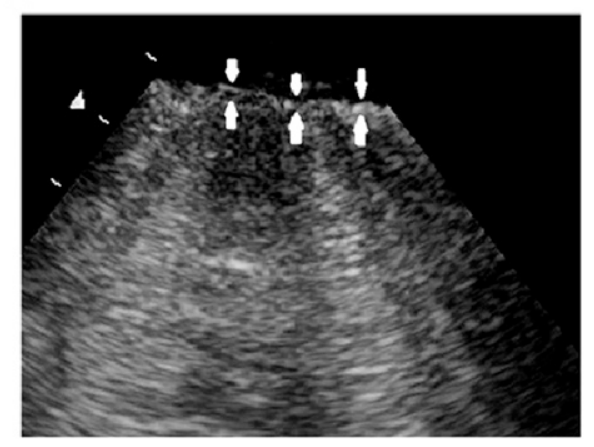

B

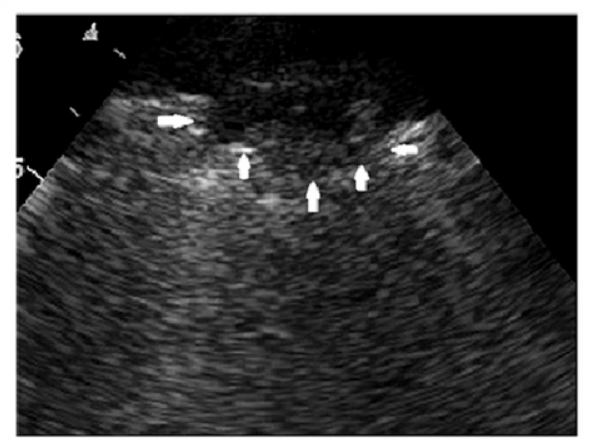

FlGURE 1. Pleural line abnormalities (A) and subpleural consolidation (B) identified with lung ultrasound in patient with COVID-19 infection. 

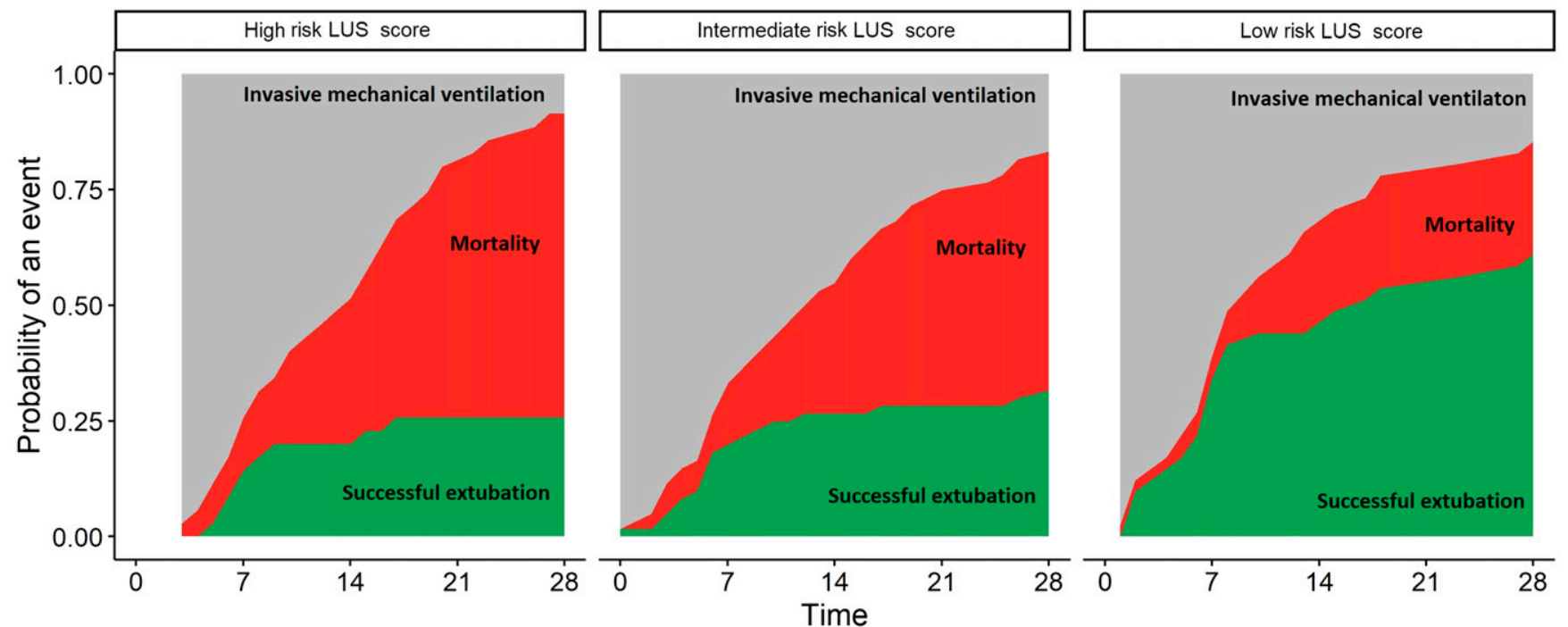

FIGURE 2. Three categories of global lung ultrasound (LUS) score and cumulative incidence of outcomes. $x$-axis: days since intubations; $y$-axis: probability of an event (extubation or death) in the population. The three facets show the risk for patients with a high risk global LUS score (left), intermediate risk (middle) and low risk global LUS score (right). Red area show the patients who died. Green area shows the patients who were successfully extubated. This figure appears in color at www.ajtmh.org.

TABLE 2

Prognostic values of global lung ultrasound (LUS) score alone and with the addition of number of areas with subpleural consolidations or pleural line abnormalities

\begin{tabular}{|c|c|c|c|c|c|c|}
\hline \multirow{4}{*}{$\begin{array}{c}\text { Outcome } \\
\text { Successful liberation } \\
\text { from mechanical } \\
\text { ventilation while } \\
\text { alive at day } 28\end{array}$} & $\frac{\text { Score }}{\text { Global LUS score }}$ & \multirow{2}{*}{$\frac{\text { AUROCC }(95 \% \mathrm{Cl})}{0.65(0.54-0.74)}$} & \multicolumn{2}{|c|}{ Cutoff } & \multirow{2}{*}{$\begin{array}{c}\text { Poor outcome } \\
68 \\
16 \\
\text { Sensitivity: } 81\end{array}$} & \multirow{2}{*}{$\begin{array}{c}\text { Good outcome } \\
28 \\
25 \\
\text { Specificity: } 47\end{array}$} \\
\hline & & & 17 & $\begin{array}{l}\geq 17 \\
<17\end{array}$ & & \\
\hline & & & 24 & $\geq 24$ & 26 & 9 \\
\hline & & & & $<24$ & 58 & 44 \\
\hline & & & & & Sensitivity: 31 & Specificity: 83 \\
\hline & + Subpleural & $0.65(0.55-0.75)$ & 19 & $\geq 19$ & 62 & 29 \\
\hline & & & & $<19$ & $\begin{array}{c}12 \\
\text { Sensitivity } 84\end{array}$ & $\begin{array}{c}20 \\
\text { Snecificity } \\
41\end{array}$ \\
\hline & & & 28 & $\geq 28$ & 25 & 7 \\
\hline & & & & $<28$ & 49 & 42 \\
\hline & & & & & Sensitivity: 32 & Specificity: 86 \\
\hline & + Pleural line & $0.61(0.51-0.71)$ & 18 & $\geq 18$ & 61 & 32 \\
\hline & abnormalities & & & $<18$ & $\begin{array}{c}13 \\
\text { Sensitivity. } 82\end{array}$ & $\begin{array}{c}17 \\
\text { Specificity. } 35\end{array}$ \\
\hline & & & 28 & $\geq 28$ & 13 & 17 \\
\hline & & & & $<28$ & 53 & 40 \\
\hline & & & & & Sensitivity: 28 & Specificity: 82 \\
\hline Mortality at day 28 & Global LUS score & $0.63(0.53-0.72)$ & 17 & $\geq 17$ & 54 & 42 \\
\hline & & & & $<17$ & $\begin{array}{c}10 \\
\text {. }\end{array}$ & 31 \\
\hline & & & 24 & $\geq 24$ & $\begin{array}{c}\text { Sensitivity: } 84 \\
23\end{array}$ & $\begin{array}{c}\text { speciticity: } 31 \\
12\end{array}$ \\
\hline & & & & $<24$ & 41 & 61 \\
\hline & & & & & Sensitivity: 35 & Specificity: 83 \\
\hline & + Subpleural & $0.61(0.51-0.71)$ & 19 & $\geq 19$ & 46 & 45 \\
\hline & consolidations & & & $<19$ & 10 & 22 \\
\hline & & & & & Sensitivity: 82 & Specificity: 32 \\
\hline & & & 28 & $\geq 28$ & 19 & 13 \\
\hline & & & & $<28$ & $\begin{array}{c}37 \\
\text { Sensitivity: } 33\end{array}$ & $\begin{array}{l}54 \\
\text { Specificity: } 81\end{array}$ \\
\hline & + Pleural line & $0.58(0.47-0.68)$ & 18 & $\geq 18$ & 46 & 47 \\
\hline & abnormalities & & & $<18$ & 10 & 20 \\
\hline & & & & & Sensitivity: 82 & Specificity: 30 \\
\hline & & & 28 & $\geq 28$ & 19 & 11 \\
\hline & & & & $<28$ & 37 & 56 \\
\hline & & & & & Sensitivity: 33 & Specificity: 83 \\
\hline
\end{tabular}

Prognostic accuracy stratified for successful liberation from invasive ventilation and mortality at day 28. Areas under the ROC curve (AUROCC) with optimal cutoff and corresponding sensitivity and specificity are shown for the global LUS score alone, and for the score in combination with subpleural consolidations and pleural line abnormalities. The $P$-value indicates the difference between the global LUS score and the score combined with either additional finding. 


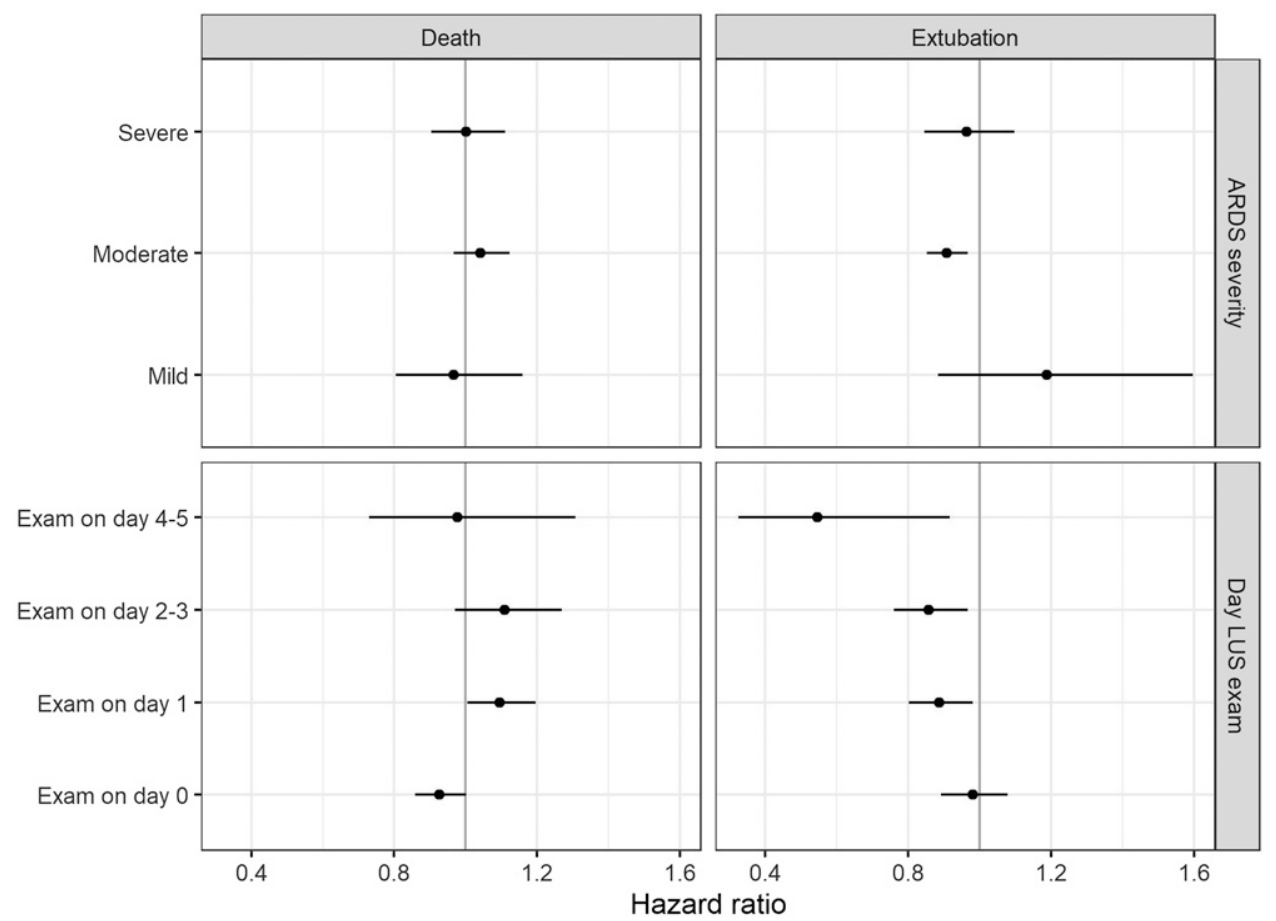

FIGURE 3. Forest plot of global lung ultrasound (LUS) score association with probability of successful liberation of invasive ventilation and death at 28 days according to acute respiratory distress syndrome (ARDS) severity and the day of examination after start invasive ventilation. $x$-axis: hazard ratio for increase of global LUS score for mortality (left) and extubation (right) based on competing risk analysis. The dots provide the point estimate and the lines the $95 \%$ confidence interval for estimated associations, stratified for predefined subgroups.

\section{DISCUSSION}

The results of this study can be summarized as follows: 1) an increasing global LUS score indicative of parenchymal damage and loss of aeration is associated with a lower likelihood of mechanical ventilation liberation during the first 28 days of invasive mechanical ventilation but not with mortality, 2) this association was independent from ARDS severity but not from timing of examination, and 3) additional LUS findings such as subpleural consolidations and pleural line abnormalities do not significantly improve the prognostic value.

LUS is attractive method for evaluation the severity of COVID-19 patients because ultrasound machines are widely available, and thus the technique can be used even in resource-limited settings. Furthermore, LUS examination at the bedside can potentially decrease or eliminate the need for transport to the radiology department, which is particularly helpful in invasively ventilated patients. Increasing global LUS scores were associated with a higher probability of requiring invasive ventilation for at least 28 days.

Severity assessment of severe COVID-19 early after invasive ventilation initiation is challenging as ventilator management can moderate the prognostic value of easily derived parameters such as the $\mathrm{P}_{\mathrm{a}} \mathrm{O}_{2} / \mathrm{FiO}_{2}{ }^{33}$ Simultaneously, the compliance of the respiratory system is low in most of these patients and has little prognostic value. ${ }^{34}$ In our study, patients who successfully extubated had a similarly low respiratory system compliance compared with patients who were not successfully extubated. The loss of aeration estimation with LUS was associated with successful extubation independent of the $\mathrm{P}_{\mathrm{a}} \mathrm{O}_{2} / \mathrm{FiO}_{2}$ categories, which are used in clinical practice for ARDS severity assessment. Hence, on the basis of these results, we think LUS can be used as an additional tool to clinical and laboratory parameters for the severity appreciation on invasively ventilated patients with COVID-19 ARDS.

We did not confirm the results of a previous study in non-COVID-19 ARDS patients that showed that a global LUS score of 16.5 was predictive for mortality. ${ }^{35}$ Because rapid extubation is predicted by less extensive parenchymal involvement, reflective of a lower degree of lung injury, we may speculate that mortality is mainly driven by the occurrence of ICU-acquired complications such as pneumonia, pulmonary embolism, ICU-acquired weakness, and the ability to endure prolonged duration of mechanical ventilation that is frequently needed for COVID-19-related ARDS. This finding is in line with a previous study that showed no association between decreased volume of well-aerated lung tissue as assessed by chest computed tomography and 30 -day mortality in critically ill patients with COVID-19 ARDS. ${ }^{36}$ Of note, that study also showed that global LUS score was a better predictor of outcome than the CT severity score. ${ }^{36}$ In non-COVID-19 ARDS, the relationship between mortality rates and the amount of not aerated areas in invasively ventilated patients is not clear either. ${ }^{37-40}$ Even though the limited duration of follow-up of 1 month may have obscured associations with longer term mortality, ${ }^{41}$ we conclude that the extent of parenchymal involvement is a poor predictor of outcome when applied to a cohort of critically ill patients requiring invasive mechanical ventilation.

We aimed to facilitate bedside estimation for the risk of adverse outcomes by identifying a cutoff for the global LUS score that could predict mortality or liberation of invasive 
ventilation in COVID-19 related ARDS patients. In contrast to previous studies that evaluated patients who did not receive invasive mechanical ventilation, ${ }^{17,19,23-25}$ we were unable to provide a single LUS value that was highly predictive of outcome. However, liberation of mechanical ventilation at 28 days was much more likely in patients with a global LUS lower than 17, whereas this was unlikely in patients with a global LUS score greater than 24. Importantly, these cutoffs are not intended to replace continuous value and are arbitrary. We prespecified a sensitivity and specificity of $80 \%$ for successful extubation, but one could argue that higher certainties are needed for clinical decision-making.

Global LUS scores obtained from exams that were performed on the first day of invasive ventilation showed to have less prognostic value compared with LUS exams that were performed after the first day. We postulate that the association between global LUS scores and outcomes are influenced by the response to invasive mechanical ventilation and/or corticosteroid treatment. In our cohort, patients examined on the first day after invasive ventilation initiation had a median LUS score of 22. This score is consistent with the results of previous study in which patients with COVID19 in the ED who required invasive ventilation had a median LUS score of $22 .^{19}$ Assessment of lung reaeration as response by computed tomography in COVID-19 patients has shown conflicting results. ${ }^{42,43}$ Assessment of the influence of ventilator management on association between global LUS scores and outcomes should be a topic for future studies.

Subpleural consolidations and pleural line abnormalities are commonly used to distinguish ARDS from cardiogenic pulmonary edema ${ }^{44}$ and are also frequently reported in patients with COVID-19-related ARDS. ${ }^{45-48}$ In theory, both of these findings can be related to the severity of COVID-19 disease. Additionally, the presence of subpleural consolidations might also indicate a pulmonary embolism. ${ }^{49}$ In terms of prognostication, both subpleural consolidations and pleural line abnormalities were found to be associated with the prognosis in COVID-19 patients outside ${ }^{15}$ and inside the hospital. ${ }^{19}$ We found more regions with subpleural consolidations in patients with a poor outcome but not more pleural line abnormalities. Nevertheless, the extent of subpleural consolidations or pleural line abnormalities were not quantitatively related to liberation of ventilation or mortality in the subset of COVID-19 patients who require intubation and mechanical ventilation. Because the global LUS score was associated with the extent of subpleural consolidations, we reason that subpleural consolidations are more related to the degree of lung aeration loss rather than to a distinct predictor of mortality or liberation of invasive ventilation.

The main strength of this study is that the global LUS score was assessed by an identical and systematic method by multiple investigators in patients who were treated in four centers in three countries. Moreover, we included only COVID-19 patients with severe respiratory failure undergoing invasive ventilation, a homogeneous group of patients that has been underrepresented in COVID-19 LUS cohorts. We accounted for competing risks and were able to distill an association with liberation of ventilation when accounting for the occurrence of mortality during the first 28 days of invasive ventilation. This study also has limitations. First, this is a retrospective study, and the indication and time point for a lung ultrasound exam was not prescribed in a protocol. Lung ultrasound exams were done as part of routine clinical practice and all performed within 5 days after start of invasive ventilation. Second, we could not assess the prognostic value of the changes in the LUS score over time as LUS exams were not performed repeatedly in the participating centers. Dynamic changes in LUS scores should be studied further as monitoring tool for reaeration of lung tissue. ${ }^{50}$ Additional studies should focus on LUS because it is an excellent technique to use in a resource-limited setting as alternative for chest radiography or chest $\mathrm{CT} .{ }^{51}$

\section{CONCLUSIONS}

The global LUS score is associated with successful liberation from invasive ventilation but not with mortality during the first 4 weeks of invasive ventilation. In patients with a low global LUS score, extubation can be expected in the first weeks of mechanical ventilation, whereas this is uncommon in patients with a high global LUS score. The extent of subpleural consolidations or pleural line abnormalities does not add prognostic value to the global LUS score in invasively ventilated patients.

Received May 14, 2021. Accepted for publication September 6, 2021.

Published online October 18, 2021.

Acknowledgments: Clinicaltrials.gov identifier: NCT04487769. The American Society of Tropical Medicine and Hygiene has waived the Open Access fee for this article due to the ongoing COVID-19 pandemic.

Authors' addresses: Charalampos Pierrakos, Amsterdam University Medical Centers-AMC, Amsterdam, The Netherlands, and Brugmann University Hospital, Université Libre de Bruxelles, Belgium, E-mail: charalampos_p@hotmail.com. Arthur Lieveld, Micah Heldeweg, Mark Haaksma, Jasper Smit, Lars Veldhuis, and Robin Walburgh Schmidt, Amsterdam University Medical Centers-VUMC, Amsterdam, The Netherlands, E-mails: a.lieveld@amsterdamumc.nl, m.heldeweg@amsterdamumc.nl, m.haaksma@amsterdamumc.nl, j.smit6@amsterdamumc.nll.i.veldhuis@amsterdamumc.nl, and r.walburgh@amsterdamumc.nl. Luigi Pisani, Amsterdam University Medical Centers-AMC, Amsterdam, The Netherlands, Miulli Regional Hospital, Acquaviva delle Fonti, Italy, and Mahidol University, Bangkok, Thailand, E-mail: luigipisani@gmail.com. Marry R. Smit, Laura A. Hagens, and Lieuwe D. Bos, Amsterdam University Medical Centers-AMC, Amsterdam, The Netherlands, E-mails: m.r. smit@amsterdamumc.nl, I.a.hagens@amsterdamumc.nl, and I.d. bos@amsterdamumc.nl. Giacomo Errico, Miulli Regional Hospital, Acquaviva delle Fonti, Italy, and Foggia University Hospital, University of Foggia, Italy, E-mail: giacomo.errico1@gmail.com. Valentina Marinelli and Francesco Murgolo, University of Bari, Bari, Italy, E-mails: v.marinelli22@gmail.com and francesco.murgolo@ uniba.it. Rachid Attou, Cristina E. David, and David De Bels, Brugmann University Hospital, Université Libre de Bruxelles, Belgium, E-mails: rachid.attou@chu-brugmann.be, cristina.david@ bordet.be, and david.debels@chu-brugmann.be. Claudio Zimatore and Salvatore Grasso, Amsterdam University Medical CentersAMC, Amsterdam, The Netherlands, and University of Bari, Bari, Italy, E-mails: claudiozimatore@gmail.com and salvatore.grasso@ uniba.it. Lucia Mirabella and Gilda Cinnella, Foggia University Hospital University of Foggia, Italy, E-mails: lucia.mirabella@unifg.it and gilda.cinnella@unifg.it. Marcus J. Schultz, Amsterdam University Medical Centers-AMC, Amsterdam, The Netherlands, Mahidol University, Bangkok, Thailand, and University of Oxford, United Kingdom, E-mail: marcus.j.schultz@gmail.com. Pieter-Roel Tuinman, Amsterdam University Medical Centers-VUMC, Amsterdam, The Netherlands, and Amsterdam Leiden IC Focussed Echograpy, E-mail: p.tuinman@amsterdamumc.nl. 
This is an open-access article distributed under the terms of the Creative Commons Attribution (CC-BY) License, which permits unrestricted use, distribution, and reproduction in any medium, provided the original author and source are credited.

\section{REFERENCES}

1. COVID-19 Dashboard by the Center for Systems Science and Engineering (CSSE) at Johns Hopkins University (JHU). Available at: https://gisanddata.maps.arcgis.com/apps/opsdash board/index.html\#/bda7594740fd40299423467b48e9ecf6? utm_source=sn\&utm_medium =referral\&utm_content=null\&utm_ campaign=BSLB_1_CA01_GL_BSLB_AWA_CA01_GL_LSGR PubH_Coronovirus_LandingPage. Accessed May 10, 2021.

2. Chen $\mathrm{N}$ et al., 2020. Epidemiological and clinical characteristics of 99 cases of 2019 novel coronavirus pneumonia in Wuhan, China: a descriptive study. Lancet 395: 507-513.

3. Guan W et al., 2020. Clinical characteristics of coronavirus disease 2019 in China. N Engl J Med 382: 1708-1720.

4. Wunsch H, 2020. Mechanical ventilation in COVID-19: interpreting the current epidemiology. Am J Respir Crit Care Med 202: $1-4$.

5. Armstrong RA, Kane AD, Kursumovic E, Oglesby FC, Cook TM, 2021. Mortality in patients admitted to intensive care with COVID-19: an updated systematic review and meta-analysis of observational studies. Anaesthesia 76: 537-548.

6. King CS et al., 2020. Outcomes of mechanically ventilated patients with COVID-19 associated respiratory failure. PLoS One 15: e0242651.

7. Angeli E et al., 2021. Prognostic value of CT integrated with clinical and laboratory data during the first peak of the COVID-19 pandemic in northern Italy: a nomogram to predict unfavorable outcome. Eur J Radiol 137: 109612.

8. Salahshour F, Mehrabineja MM, Nassiri Toosi M, Gity M, Ghanaati $\mathrm{H}$, Shakiba M, Nosrat Sheybani S, Komaki H, Kolahi S, 2021. Clinical and chest CT features as a predictive tool for COVID-19 clinical progress: introducing a novel semiquantitative scoring system. Eur Radiol 15: 1-11.

9. Jeong YJ, Nam BD, Yoo JY, Kim KI, Kang H, Hwang JH, Kim $\mathrm{YH}$, Lee KS, 2021. Prognostic implications of CT feature analysis in patients with COVID-19: a nationwide cohort study. $J$ Korean Med Sci 36: e51.

10. Bello G, Blanco P, 2019. Lung ultrasonography for assessing lung aeration in acute respiratory distress syndrome: a narrative review. J Ultrasound Med 38: 27-37.

11. Bouhemad B, Brisson H, Le-Guen M, Arbelot C, Lu Q, Rouby JJ, 2011. Bedside ultrasound assessment of positive endexpiratory pressure-induced lung recruitment. Am J Respir Crit Care Med 183: 341-347.

12. Mongodi S, Bouhemad B, Orlando A, Stella A, Tavazzi G, Via G, lotti GA, Brasch A, Mojoli F, 2017. Modified lung ultrasound score for assessing and monitoring pulmonary aeration. Ultraschall Med 38: 530-537.

13. Pisani $L$ et al., 2019. The diagnostic accuracy for ARDS of global versus regional lung ultrasound scores - a post hoc analysis of an observational study in invasively ventilated ICU patients. Intensive Care Med Exp 25(Suppl 1): 44.

14. Tierney DM, Boland LL, Overgaard JD, Huelster JS, Jorgenson A, Normington JP, Melamed RR, 2018. Pulmonary ultrasound scoring system for intubated critically ill patients and its association with clinical metrics and mortality: a prospective cohort study. J Clin Ultrasound 46: 14-22.

15. Mafort TT, Rufino R, da Costa $\mathrm{CH}$, da Cal MS, Monnerat LB, Litrento PF, Parra L, Marinho A, Lopes AJ, 2021. One-month outcomes of patients with SARS-CoV-2 infection and their relationships with lung ultrasound signs. Ultrasound $J$ 13: 19.

16. de Alencar J, Marchini J, Marino LO, da Costa Ribeiro SC, Bueno CG, da Cunha VP, Lazar Neto F, Brandão Neto RA, Souza HP, 2021. Lung ultrasound score predicts outcomes in COVID-19 patients admitted to the emergency department. Ann Intensive Care 11: 6.

17. Secco $G$ et al., 2021. Lung ultrasound presentation of COVID-19 patients: phenotypes and correlations. Intern Emerg Med 16: 1317-1327.
18. Arthur WE et al., 2021. Assessing COVID-19 pneumonia-clinical extension and risk with point-of-care ultrasound: a multicenter, prospective, observational study. J Am Coll Emerg Physicians Open 2: 1-12.

19. Lichter $Y$ et al., 2020. Lung ultrasound predicts clinical course and outcomes in COVID-19 patients. Intensive Care Med 46: 1873-1883.

20. Kumar A, et al., 2021. Lung ultrasound findings in patients hospitalized with COVID-19. J Ultrasound Med (In press). Available at: https://doi.org/10.1002/jum.15683.

21. Szekely $Y$ et al., 2021. The predictive role of combined cardiac and lung ultrasound in coronavirus disease 2019. J Am Soc Echocardiogr 34: 642-652.

22. Yasukawa K, Minami T, Boulware DR, Shimada A, Fischer EA, 2021. Point-of-care lung ultrasound for COVID-19: findings and prognostic implications from 105 consecutive patients. J Intensive Care Med 36: 334-342.

23. Ji $L$ et al., 2020. Prognostic value of bedside lung ultrasound score in patients with COVID-19. Crit Care 24: 700.

24. Rubio-Gracia J et al., 2021. Point-of-care lung ultrasound assessment for risk stratification and therapy guiding in COVID-19 patients. A prospective non-interventional study. Eur Respir J 36: 334-342.

25. Tombini $V$ et al., 2021. Risk stratification in COVID-19 pneumonia - determining the role of lung ultrasound. Ultraschall Med. doi:10.1055/a-1344-4715.

26. Casella $F$ et al., 2021. Lung ultrasonography: a prognostic tool in non-ICU hospitalized patients with COVID-19 pneumonia. Eur J Intern Med 85: 34-40.

27. Duclos G et al., 2021. Can thoracic ultrasound on admission predict the outcome of critically ill patients with SARS-CoV-2? A French multi-centric ancillary retrospective study. Adv Ther 38: 2599-2612.

28. Schultz MJ, Sivakorn C, Dondorp AM, 2020. Challenges and opportunities for lung ultrasound in novel coronavirus disease (COVID-19). Am J Trop Med Hyg 102: 1162-1163.

29. Kruisselbrink R, Chan V, Cibinel GA, Abrahamson S, Goffi A, 2017. I-AIM (Indication, Acquisition, Interpretation, Medical Decision-making) framework for point of care lung ultrasound. Anesthesiology 127: 568-582.

30. Mojoli F, Bouhemad B, Mongodi S, Lichtenstein D, 2019. Lung ultrasound for critically ill patients. Am J Respir Crit Care Med 199: 701-714.

31. Ranieri VM, Rubenfeld GD, Thompson BT, Ferguson ND, Caldwell E, Fan E, Camporota L, Slutsky AS, 2012. Acute respiratory distress syndrome: the Berlin Definition. JAMA 307: 2526-2533.

32. Safari S, Baratloo A, Elfil M, Negida A, 2016. Evidence based emergency medicine; part 5 receiver operating curve and area under the curve. Emergency (Tehran) 4: 111-113.

33. Palanidurai S, Phua J, Chan YH, Mukhopadhyay A, 2021. P/FP ratio: incorporation of PEEP into the $\mathrm{PaO} 2 / \mathrm{FiO} 2$ ratio for prognostication and classification of acute respiratory distress syndrome. Ann Intensive Care 11: 124.

34. Botta $M$ et al., 2021. Ventilation management and clinical outcomes in invasively ventilated patients with COVID-19 (PRoVENT-COVID): a national, multicentre, observational cohort study. Lancet Respir Med 9: 139-148.

35. Zhao Z, Jiang L, Xi X, Jiang Q, Zhu B, Wang M, Xing J, Zhang $D$, 2015. Prognostic value of extravascular lung water assessed with lung ultrasound score by chest sonography in patients with acute respiratory distress syndrome. BMC Pulm Med 15: 98.

36. Heldeweg $M$ et al., 2021. Lung ultrasound and computed tomography to monitor COVID-19 pneumonia in critically ill patients: a two-center prospective cohort study. Intensive Care Med Exp 9: 1.

37. Gattinoni L et al., 2006. Lung recruitment in patients with the acute respiratory distress syndrome. $N$ Engl $J$ Med 354: 1775-1786.

38. Caironi $P$ et al., 2010. Lung opening and closing during ventilation of acute respiratory distress syndrome. Am J Respir Crit Care Med 181: 578-586.

39. de Matos GF et al., 2012. How large is the lung recruitability in early acute respiratory distress syndrome: a prospective case 
series of patients monitored by computed tomography. Crit Care 16: R4.

40. Camporota L et al., 2019. Lung recruitability in severe acute respiratory distress syndrome requiring extracorporeal membrane oxygenation. Crit Care Med 47: 1177-1183.

41. Gamberini L et al., 2020. Factors influencing liberation from mechanical ventilation in coronavirus disease 2019: multicenter observational study in fifteen Italian ICUs. J Intensive Care 8: 80.

42. Pan C, Chen L, Lu C, Zhang W, Xia JA, Sklar MC, Du B, Brochard L, Qiu H, 2020. Lung recruitability in COVID-19associated acute respiratory distress syndrome: a singlecenter observational study. Am J Respir Crit Care Med 201: 1294-1297.

43. Ball L et al., 2021. Computed tomography assessment of PEEPinduced alveolar recruitment in patients with severe COVID19 pneumonia. Crit Care 25: 81.

44. Volpicelli $G$ et al., 2012. International evidence-based recommendations for point-of-care lung ultrasound. Intensive Care Med 38: 577-591.

45. Peng QY et al., 2020. Findings of lung ultrasonography of novel corona virus pneumonia during the 2019-2020 epidemic. Intensive Care Med 46: 849-850.
46. Lomoro P, Verde F, Zerboni F, Simonetti I, Borghi C, Fachinetti C, Natalizi A, Martegani A, 2020. COVID-19 pneumonia manifestations at the admission on chest ultrasound, radiographs, and CT: single-center study and comprehensive radiologic literature review. Eur J Radiol Open 7: 100231.

47. Yasukawa K, Minami T, 2020. Point-of-care lung ultrasound findings in patients with COVID-19 pneumonia. Am J Trop Med Hyg 102: 1198-1202.

48. Haaksma ME et al., 2020. Lung ultrasound findings in patients with novel SARS-CoV-2. ERJ Open Res 6: 00238-02020. doi: 10.1183/23120541.00238-2020.

49. Zotzmann V, Lang CN, Bamberg F, Bode C, Staudacher DL, 2020. Are subpleural consolidations indicators for segmental pulmonary embolism in COVID-19? Intensive Care Med 46: $1109-1110$.

50. Pierrakos $C$, Attou R, lesu E, Baelongandi $H$, Honore PM, Bos L, Schultz MJ, De Bels D, 2020. Case report: lung ultrasound for the guidance of adjunctive therapies in two invasively ventilated patients with COVID-19. Am J Trop Med Hyg 103: 1978-1982.

51. Schultz MJ, Sivakorn C, Dondorp AM, 2020. Challenges and opportunities for lung ultrasound in novel coronavirus disease (COVID-19). Am J Trop Med Hyg 102: 1162-1163. 